\title{
Point counting on K3 surfaces and an application concerning real and complex multiplication
}

\author{
Andreas-Stephan Elsenhans and Jörg Jahnel
}

\begin{abstract}
We report on our project to find explicit examples of K3 surfaces having real or complex multiplication. Our strategy is to search through the arithmetic consequences of RM and CM. In order to do this, an efficient method is needed for point counting on surfaces defined over finite fields. For this, we describe algorithms that are $p$-adic in nature.
\end{abstract}

\section{Introduction}

Let $X$ be a quasi-projective variety over a finite field $\mathbb{F}_{q}$ of characteristic $p>0$. One of the most natural questions concerning the arithmetic of $X$ is certainly to pinpoint the number of points that $X$ has over its base field $\mathbb{F}_{q}$. For example, let $X:=V(f) \subset \mathbf{P}^{N}$ be given for $f \in \mathbb{F}_{q}\left[T_{0}, \ldots, T_{N}\right]$ a homogeneous polynomial. Then this means just to count the number of solutions in $\mathbb{F}_{q}^{N+1}$ of the equation $f\left(T_{0}, \ldots, T_{N}\right)=0$, ignoring $(0, \ldots, 0)$ and up to scaling.

More generally, one looks for the sequence $\left(\# X\left(\mathbb{F}_{q^{i}}\right)\right)_{i \geqslant 1}$ of integers associated with $X$ that is composed of the numbers of points on $X$ that are defined over the extension fields $\mathbb{F}_{q^{i}}$. This sequence is of practical as well as theoretical interest. For example, it has been known since Artin wrote his $\mathrm{PhD}$ thesis $[2, \S 22]$ that it is wise to form the generating function

$$
Z_{X}(t):=\exp \left(\sum_{i=1}^{\infty} \# X\left(\mathbb{F}_{q^{i}}\right) \frac{t^{i}}{i}\right)
$$

which is called the zeta function of the variety $X$. In fact, $Z_{X}$ is always a rational function. According to [24, Exp. XV, $\S 3, \operatorname{nos} 2$ and 3], it is the alternating product of the Weil polynomials $\chi_{i}$ associated with $X$, the characteristic polynomials of the operation of the geometric Frobenius Frob on the $l$-adic cohomology vector spaces $H_{c, \text { ét }}^{i}\left(X_{\overline{\mathbb{F}}_{q}}, \mathbb{Q}_{l}\right)$ with compact support,

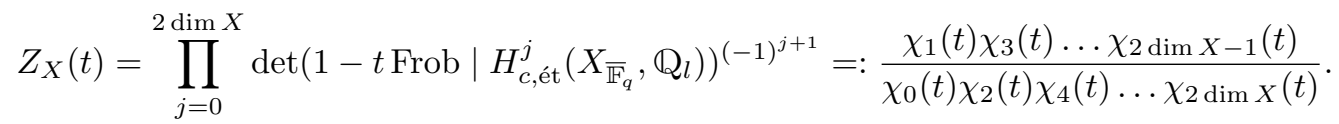

Moreover, at least when $X$ is proper and smooth, the Weil polynomials $\chi_{j}$ have remarkable properties. For example, as was conjectured by Weil and proven by Deligne in $[\mathbf{8}$, Théorème (1.6)] and [10, Corollaire (3.3.9)], every complex root of $\chi_{j}$ is of absolute value $q^{j / 2}$. In particular, no cancellations occur in formula (2).

Assume now that the geometry of the variety $X_{\overline{\mathbb{F}}_{q}}$ is well understood, by which we mean that at least all its $l$-adic Betti numbers are known. This is usually the case in practice, as the varieties considered are curves of known genus, abelian varieties of known dimension, nonsingular complete intersections of known multidegree, K3 surfaces, etc. From the algorithmic

Received 6 February 2016.

2010 Mathematics Subject Classification 14J28 (primary), 11G15, 11Y16, 11M38 (secondary).

Contributed to the Twelfth Algorithmic Number Theory Symposium (ANTS-XII), Kaiserslautern, Germany, 29 August-2 September 2016. 
point of view, formula (2) then shows that it is easy to calculate $\# X\left(\mathbb{F}_{q^{i}}\right)$ for some very large $i$, provided that $\# X\left(\mathbb{F}_{q}\right), \# X\left(\mathbb{F}_{q^{2}}\right), \ldots, \# X\left(\mathbb{F}_{q^{k}}\right)$ have been found. Here, the bound $k$ only depends on the geometry of $X_{\overline{\mathbb{F}}_{q}}$. Indeed, once $k$ is properly chosen, (1) and (2) allow the computation of all the coefficients of the polynomials $\chi_{j}$ from the numbers of points known. Then pinpointing $\# X\left(\mathbb{F}_{q^{i}}\right)$ is just a matter of calculating the $i$ th power series coefficient of $\sum_{j}(-1)^{j+1} \log \chi_{j}$.

Unfortunately, for most non-trivial types of varieties, the bound $k$ turns out to be too large, such that it is not feasible to count $\# X\left(\mathbb{F}_{q}\right), \# X\left(\mathbb{F}_{q^{2}}\right), \ldots, \# X\left(\mathbb{F}_{q^{k}}\right)$ in a naive way, even for medium-sized values of $q$. For instance, when $X$ is a K3 surface, things are relatively simple. In fact, $\chi_{1}(t)=\chi_{3}(t)=1, \chi_{0}(t)=1-t, \chi_{4}(t)=1-q^{2} t$, and only $\chi_{2}$ varies, which is of degree 22 . Nevertheless, one may only hope that $k=10$ is sufficient. It is known that there are cases where $k$ has to be chosen larger [17, Table 6].

\subsection{Our motivation. Real and complex multiplication for K3 surfaces}

Our motivation comes from projective varieties $X$ that are defined over a number field $K$. As was first noticed by van Luijk, from point counts on the reductions $X_{\mathfrak{p}_{1}}, X_{\mathfrak{p}_{2}}$ modulo two primes $[\mathbf{1 6}, \mathbf{1 9}, \mathbf{3 1}, \mathbf{3 2}]$ of good reduction or sometimes only one [18], one may determine the geometric Néron-Severi rank of $X$. This applies well even to varieties of general type [15], but a non-trivial case is provided already by K3 surfaces.

The very basic idea behind van Luijk's method is that, in the case of a K3 surface, $\operatorname{rk} \operatorname{Pic} X_{\overline{\mathbb{F}}_{\mathfrak{p}}}$ is always even and one has $\operatorname{rk} \operatorname{Pic} X_{\overline{\mathbb{F}}_{\mathfrak{p}}} \geqslant \operatorname{rk} \operatorname{Pic} X_{\bar{K}}$. Moreover, one hopes to find a prime such that $\operatorname{rkPic} X_{\overline{\mathbb{F}}_{\mathfrak{p}}}$ is actually equal to $\operatorname{rkPic} X_{\bar{K}}$ or $\operatorname{rkPic} X_{\bar{K}}+1$. As was observed by Charles [5], the existence of such primes is related to whether $X$ has real multiplication or not. More precisely, existence is provided unless $X$ has real multiplication and $\left(22-\operatorname{rk} \operatorname{Pic} X_{\bar{K}}\right) /[E: \mathbb{Q}]$ is odd, for $E$ the endomorphism field.

We say that a K3 surface $X$ has real or complex multiplication when the endomorphism algebra of the transcendental part $T \subset H^{2}(X(\mathbb{C}), \mathbb{Q})$, considered as a pure $\mathbb{Q}$-Hodge structure, is strictly larger than $\mathbb{Q}$. It is then either a totally real field or a CM field [41, Theorems 1.6(a) and 1.5.1]. Starting from this definition, however, it seems hard to conclude anything for concrete examples. One may deduce $[\mathbf{2 3}]$ at least that having RM or CM is a property of positive codimension in the analytic moduli space of K3 surfaces of fixed Picard rank (unless the Picard rank is 20, in which case every K3 surface has CM). Thus, having RM or CM should be thought of as being exceptional.

In particular, it seems that one does not find examples of RM or CM surfaces just by accident. Our approach is therefore to perform our searches through the arithmetic consequences of RM and CM. These include that, for all primes $\mathfrak{p}$ that are (at least partially) inert in $E$, the reduction $X_{\mathfrak{p}}$ is non-ordinary. That is, that one has $\# X_{\mathfrak{p}}\left(\mathbb{F}_{\mathfrak{p}}\right) \equiv 1(\bmod p)$ for $p$ the prime number below $\mathfrak{p}$. Moreover, the transcendental factors of the corresponding Weil polynomials are all partially split over the same field, the endomorphism field $E$. Compare the beginning of $\S 5$ for a more precise statement. In order to detect the very few surfaces showing such a behaviour within a large family, efficient methods for point counting are required.

\subsection{Harvey's $p$-adic method for counting points}

The most efficient methods for counting points on a variety over a finite field that are known today are $p$-adic in nature. One of them was originally developed by Kedlaya in [28]. Kedlaya's point of view was cohomological. This means that he computed the characteristic polynomial of the Frobenius operation on the Monsky-Washnitzer cohomology, a well-behaved cohomology theory with $p$-adic coefficients for affine varieties in characteristic $p$. This approach seems to be very natural. It is, however, still limited to curves, and in fact to particular types of them. Satoh's $p$-adic algorithm [35] for ordinary elliptic curves could perhaps be seen as a predecessor of this method. 
On the other hand, as was shown by Harvey [26], an entirely elementary approach is possible, as well as a generalisation to arbitrary schemes of finite type over a finite field. Harvey's method is, at least partially, based on earlier ideas. We are most likely unaware of some of its predecessors, but the work of Wan $[\mathbf{3 9}, \mathbf{4 0}]$, as well as that of Lauder and Wan [30], certainly should be mentioned. Dwork $[\mathbf{1 3}, \mathbf{1 4}]$ is probably the very first to whom the ideas behind $[\mathbf{2 6}]$ may be traced back.

We report on a variation of Harvey's p-adic method for double covers of the projective space, which we implemented as far as required for the needs of our search for K3 surfaces of degree 2 having RM or CM. Although never mentioned in print, this variation was certainly known, or at least obvious, to Harvey before. Generally speaking, it seems that the algorithm described in $[\mathbf{2 6}]$, although general in theory, may in practice be implemented only in a form specialised to particular types of varieties. Our code is available within magma [4], from version 2.22 onwards.

\section{Counting points on varieties over finite fields: elementary methods}

Let us assume that $X \subset \mathbf{A}_{\mathbb{F}_{q}}^{N}$ is an affine hypersurface; that is, that $X=V(f)$ for an arbitrary polynomial $f \in \mathbb{F}_{q}\left[T_{1}, \ldots, T_{N}\right]$. At least in theory, this is not a serious restriction as every variety over a base field is birationally equivalent to a hypersurface $[\mathbf{2 5}$, Chapter I, Proposition 4.9]; cf. [26, $\S 1.3]$. In practice, of course, excessively complicated transformations are undesirable, as they obviously reduce the speed of the method, even if only by a constant factor.

2.1 (Determination of the points). The most naive approach to point counting is certainly to determine all the points. If, for whatever application, the actual points are required, then there is no alternative to such a method. Moreover, to have some code available that realises a naive approach is useful for testing more advanced algorithms.

In order to determine the $\mathbb{F}_{q^{i}}$-rational points on $X$, one has to run an iterated loop over all $(N-1)$-tuples $\left(x_{1}, \ldots, x_{N-1}\right) \in \mathbb{F}_{q^{i}}^{N-1}$. The roots in $\mathbb{F}_{q^{i}}$ of a univariate polynomial $g$ have to be found each time, which just means computing $\operatorname{gcd}\left(g, T^{q^{i}}-T\right)$. Thus, the complexity of determining the $\mathbb{F}_{q^{i}}$-rational points is essentially $O\left(q^{(N-1) i}\right)$.

REMARK 2.2. When $X$ is defined over $\mathbb{F}_{q}$, but points over $\mathbb{F}_{q^{i}}$ for $i>1$ are sought, one may gain a factor of $i$ by dealing with Frobenius orbits instead of points.

2.3 (FFT point counting). Assume that $X$ is given by a decoupled polynomial $f$; that is, that $f=f_{1}\left(T_{1}, \ldots, T_{M}\right)+f_{2}\left(T_{M+1}, \ldots, T_{N}\right)$ for some $1<M<N$. Then $\# X\left(\mathbb{F}_{q^{i}}\right)=\left(c_{1} * c_{2}\right)(0)$ in terms of the two counting functions $c_{1}$ and $c_{2}$, given by

$$
c_{1}(c):=\#\left\{\left(x_{1}, \ldots, x_{M}\right) \in \mathbb{F}_{q^{i}}^{M} \mid f_{1}\left(x_{1}, \ldots, x_{M}\right)=c\right\}
$$

and

$$
c_{2}(c):=\#\left\{\left(x_{M+1}, \ldots, x_{N}\right) \in \mathbb{F}_{q^{i}}^{N-M} \mid f_{2}\left(x_{M+1}, \ldots, x_{N}\right)=c\right\} .
$$

In order to compute $\# X\left(\mathbb{F}_{q^{i}}\right)$, one first compiles look-up tables for $c_{1}$ and $c_{2}$ and then calculates $\left(c_{1} * c_{2}\right)(0)$, according to the definition. The complexity of this method of counting the $\mathbb{F}_{q^{i}}$ rational points is $O\left(q^{\max \{M, N-M\} i}\right)$.

Similarly, for the double cover of $\mathbf{A}^{N}$ given by $W^{2}=f$, one has

$$
\# X\left(\mathbb{F}_{q^{i}}\right)=q^{i N}+\sum_{c \in \mathbb{F}_{q^{i}}} \chi(c) \cdot\left(c_{1} * c_{2}\right)(c),
$$

where $\chi: \mathbb{F}_{q^{i}} \rightarrow\{-1,0,1\}$ denotes the quadratic character. The convolution $c_{1} * c_{2}$ then has to be computed using the FFT method ([22, Sätze 20.2 and 20.3] or [6, Theorem 32.8]). Here, 
again, the complexity is essentially $O\left(q^{\max \{M, N-M\} i}\right)$, with an additional log factor coming from the Fourier transforms. See [16, Algorithm 17] for more details on this method.

Example 2.4 (cf. [21, Example 3.13]). Consider the K3 surface $X$ of degree 2 over $\mathbb{F}_{7}$, given by

$$
\begin{aligned}
W^{2}= & 6 T_{0}^{6}+6 T_{0}^{5} T_{1}+2 T_{0}^{5} T_{2}+6 T_{0}^{4} T_{1}^{2}+5 T_{0}^{4} T_{2}^{2}+5 T_{0}^{3} T_{1}^{3} \\
& +T_{0}^{2} T_{1}^{4}+6 T_{0} T_{1}^{5}+5 T_{0} T_{2}^{5}+3 T_{1}^{6}+5 T_{2}^{6} .
\end{aligned}
$$

The polynomial on the right-hand side does not contain any monomial involving both $T_{1}$ and $T_{2}$. Thus, over the affine plane $\mathbf{P}_{\overline{\mathbb{F}}_{7}}^{2} \backslash V\left(T_{0}\right)$, the double cover $X$ is given by a decoupled polynomial. The numbers of points over $\mathbb{F}_{7}, \ldots, \mathbb{F}_{7^{10}}$ are

$$
\begin{gathered}
60,2488,118587,5765828,282498600,13841656159,678225676496, \\
33232936342644,1628413665268026,79792266679604918 .
\end{gathered}
$$

Using the FFT method, it took about 2 hours of CPU time and required approximately $5 \mathrm{~GB}$ of memory to compute these numbers on an AMD 248 Opteron processor running at $2.2 \mathrm{GHz}$.

2.5 (Making étale cohomology explicit). For a general variety, it is probably a tough ask to make its $l$-adic cohomology vector spaces explicit, including the Frobenius operation on them. There are, however, certain special types of varieties for which this is possible. For example, for an elliptic curve, one has $H_{\text {ét }}^{1}\left(X_{\overline{\mathbb{F}}_{q}}, \mathbb{Q}_{l}\right) \cong \varliminf_{n} E\left(\overline{\mathbb{F}}_{q}\right)\left[l^{n}\right] \otimes_{\mathbb{Z}_{l}} \mathbb{Q}_{l}$, which is the starting point of Schoof's algorithm [36].

A different kind are those varieties for which all the cycle maps [9, Cycle, $\S 2.2 .10]$

$$
\mathrm{cl}: \mathrm{CH}^{j}\left(X_{\overline{\mathbb{F}}_{q}}\right) \otimes_{\mathbb{Z}} \mathbb{Q}_{l} \longrightarrow H_{\text {ét }}^{2 j}\left(X_{\overline{\mathbb{F}}_{q}}, \mathbb{Q}_{l}(j)\right)
$$

are surjective and, moreover, $H_{\text {ét }}^{j}\left(X_{\overline{\mathbb{F}}_{q}}, \mathbb{Q}_{l}\right)=0$ for every odd integer $j$. These assumptions are fulfilled, for instance, for surfaces that are geometrically rational.

A case for which this approach is implemented in magma is that of a cubic surface. Here, $\mathrm{CH}^{1}\left(X_{\overline{\mathbb{F}}_{q}}\right)=\operatorname{Pic}\left(X_{\overline{\mathbb{F}}_{q}}\right) \cong \mathbb{Z}^{7}$ is generated by 27 lines. One has to determine the lines by a Gröbner base calculation. From the operation of Frob on the lines, one deduces the operation on $H_{\text {ét }}^{2}\left(X_{\overline{\mathbb{F}}_{q}}, \mathbb{Q}_{l}(1)\right)$.

\section{EXAMPLE 2.6.}

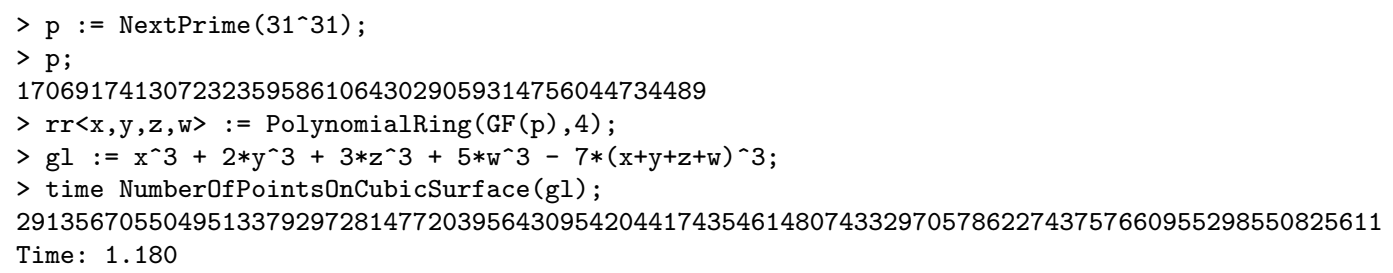

REMARK 2.7. The list of methods given in this section is by no means meant to be exhaustive. There is at least one further idea, which we can mention only in passing, namely the use of a fibration. For more information, we advise the reader to consult the articles [29] of Lauder and [34] of Pancratz and Tuitman.

\section{Harvey's p-adic method}

As above, we assume that $\mathbb{F}_{q}$ is a finite field of characteristic $p>0$. The method is $p$-adic in nature. It finds an approximation of $\# X\left(\mathbb{F}_{q^{i}}\right)$ with respect to the $p$-adic valuation. Thus, 
in order to pinpoint $\# X\left(\mathbb{F}_{q^{i}}\right)$ exactly, one needs, in addition, an estimate for the number of points.

\section{Characteristic functions}

Notation 3.1. (i) We continue to assume that $X$ is an affine hypersurface. However, we now suppose that $X:=V(f) \cap \mathbb{G}_{m, \mathbb{F}_{q}}^{N} \subset \mathbb{G}_{m, \mathbb{F}_{q}}^{N}$ is a subscheme of an affine torus. Again, this restriction is not serious as $\mathbf{A}_{\mathbb{F}_{q}}^{N}$ is a finite union of affine tori. For varieties more general than hypersurfaces, one might, in principle, simplify the situation by iteratively projecting away from points; cf. $[\mathbf{2 6}, \S 1.3]$.

(ii) For every natural number $i$, let us denote by $\mu_{q^{i}-1}$ the group of all $\left(q^{i}-1\right)$ th roots of unity and write $\mathbb{Z}_{q^{i}}$ for the integer ring of the local field $\mathbb{Q}_{p}\left(\mu_{q^{i}-1}\right)$, which is an unramified extension of $\mathbb{Q}_{p} . \mathbb{Z}_{q^{i}}$ is thus a complete discrete valuation ring with residue field $\mathbb{F}_{q^{i}}$. Furthermore, $\mu_{q^{i}-1} \subset \mathbb{Z}_{q^{i}}$ is bijectively mapped onto $\mathbb{F}_{q^{i}}^{*}$ under the residue map

$$
\pi=\pi_{i}: \mathbb{Z}_{q^{i}} \rightarrow \mathbb{F}_{q^{i}} .
$$

This is, of course, an instance of taking Teichmüller representatives.

(iii) Moreover, one has $\mathbb{Z}_{q^{i}} \subseteq \mathbb{Z}_{q^{i^{\prime}}}$ if and only if $\mathbb{F}_{q^{i}} \subseteq \mathbb{F}_{q^{i^{\prime}}}$. In this case, we introduce the quasi norm map

$$
\begin{aligned}
N_{\mathbb{Z}_{q^{i}} / \mathbb{Z}_{q^{i}}}: \mathbb{Z}_{q^{i}}\left[T_{1}, \ldots, T_{N}\right] & \longrightarrow \mathbb{Z}_{q^{i}}\left[T_{1}, \ldots, T_{N}\right], \\
g & \mapsto g \cdot g^{\left(q^{i}\right)} \cdot g^{\left(q^{2 i}\right)} \cdot \ldots \cdot g^{\left(q^{i^{\prime}-i}\right)},
\end{aligned}
$$

for $g^{(n)}$ the quasi power, given by $g^{(n)}\left(T_{1}, \ldots, T_{N}\right):=g\left(T_{1}^{n}, \ldots, T_{N}^{n}\right)$.

The quasi norm is compatible with the usual norm map $\mathrm{N}_{\mathbb{F}_{q^{\prime}} / \mathbb{F}_{q^{i}}}: \mathbb{F}_{q^{i^{\prime}}} \rightarrow \mathbb{F}_{q^{i}}$ between the finite residue fields in the sense that, for arbitrary $g \in \mathbb{Z}_{q^{i}}\left[T_{1}, \ldots, T_{N}\right]$ and $z_{1}, \ldots, z_{N} \in \mathbb{Z}_{q^{i^{\prime}}}$, one has $N_{\mathbb{Z}_{q^{i}} / \mathbb{Z}_{q^{i}}} g\left(z_{1}, \ldots, z_{N}\right) \in \mathbb{Z}_{q^{i}}$ and

$$
\pi_{i}\left(N_{\mathbb{Z}_{q^{i}} / \mathbb{Z}_{q^{i}}} g\left(z_{1}, \ldots, z_{N}\right)\right)=\mathrm{N}_{\mathbb{F}_{q^{i^{i}}} / \mathbb{F}_{q^{i}}}\left(\bar{g}\left(\pi_{i^{\prime}}\left(z_{1}\right), \ldots, \pi_{i^{\prime}}\left(z_{N}\right)\right)\right) .
$$

Here, $\bar{g} \in \mathbb{F}_{q^{i}}\left[T_{1}, \ldots, T_{N}\right]$ denotes the reduction of $g$ modulo $(p)$.

(iv) We choose a lift $F \in \mathbb{Z}_{q}\left[T_{1}, \ldots, T_{N}\right]$ of $f$.

Definition 3.2. We call a function $\Phi: \mu_{q^{i}-1}^{N} \rightarrow \mathbb{Z}_{q}$ a characteristic function of $X\left(\mathbb{F}_{q^{i}}\right)$ modulo $p^{l}$ if, for every $\left(z_{1}, \ldots, z_{N}\right) \in \mu_{q^{i}-1}^{N}$,

$$
\Phi\left(z_{1}, \ldots, z_{N}\right) \equiv 0 \quad\left(\bmod p^{l}\right) \quad \text { if }\left(\pi\left(z_{1}\right), \ldots, \pi\left(z_{N}\right)\right) \notin X\left(\mathbb{F}_{q^{i}}\right)
$$

and

$$
\Phi\left(z_{1}, \ldots, z_{N}\right) \equiv 1 \quad\left(\bmod p^{l}\right) \quad \text { if }\left(\pi\left(z_{1}\right), \ldots, \pi\left(z_{N}\right)\right) \in X\left(\mathbb{F}_{q^{i}}\right) .
$$

Proposition 3.3. Let a be a positive integer such that $a(q-1) \geqslant l$ and $i \in \mathbb{N}$ be arbitrary. Then

$$
\begin{aligned}
\Phi & :=\left(1-N_{\mathbb{Z}_{q^{i}} / \mathbb{Z}_{q}} F^{a(q-1)}\right)^{l} \\
& =1+\sum_{k=1}^{l}(-1)^{k}\left(\begin{array}{l}
l \\
k
\end{array}\right) N_{\mathbb{Z}_{q^{i}} / \mathbb{Z}_{q}} F^{k a(q-1)}
\end{aligned}
$$

is a characteristic function of $X\left(\mathbb{F}_{q^{i}}\right)$ modulo $p^{l}$. 
Proof. By definition, $\left(\pi\left(z_{1}\right), \ldots, \pi\left(z_{N}\right)\right) \in X\left(\mathbb{F}_{q^{i}}\right)$ if and only if $f\left(\pi\left(z_{1}\right), \ldots, \pi\left(z_{N}\right)\right)=0 \in \mathbb{F}_{q^{i}}$, which is equivalent to $\mathrm{N}_{\mathbb{F}_{q^{i}} / \mathbb{F}_{q}}\left(f\left(\pi\left(z_{1}\right), \ldots, \pi\left(z_{N}\right)\right)\right)=0 \in \mathbb{F}_{q}$ and

$$
N_{\mathbb{Z}_{q^{i}} / \mathbb{Z}_{q}} F\left(z_{1}, \ldots, z_{N}\right) \equiv 0 \quad(\bmod p) .
$$

If this is true then $N_{\mathbb{Z}_{q^{i}} / \mathbb{Z}_{q}} F^{a(q-1)}\left(z_{1}, \ldots, z_{N}\right) \equiv 0\left(\bmod p^{a(q-1)}\right)$, hence the same modulo $p^{l}$, which implies $1-N_{\mathbb{Z}_{q^{i}} / \mathbb{Z}_{q}} F^{a(q-1)}\left(z_{1}, \ldots, z_{N}\right) \equiv 1\left(\bmod p^{l}\right)$, and

$$
\left(1-N_{\mathbb{Z}_{q^{i}} / \mathbb{Z}_{q}} F^{a(q-1)}\left(z_{1}, \ldots, z_{N}\right)\right)^{l} \equiv 1 \quad\left(\bmod p^{l}\right) .
$$

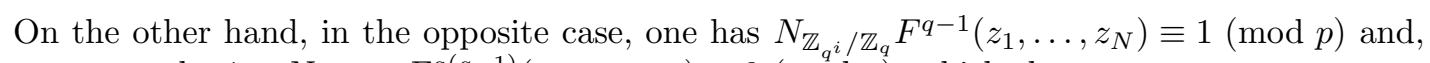
consequently, $1-N_{\mathbb{Z}_{q^{i}} / \mathbb{Z}_{q}} F^{a(q-1)}\left(z_{1}, \ldots, z_{N}\right) \equiv 0(\bmod p)$, which shows

$$
\left(1-N_{\mathbb{Z}_{q^{i}} / \mathbb{Z}_{q}} F^{a(q-1)}\left(z_{1}, \ldots, z_{N}\right)\right)^{l} \equiv 0 \quad\left(\bmod p^{l}\right),
$$

as required.

Remarks 3.4. (i) According to the definition, if $\Phi$ is a characteristic function of $X\left(\mathbb{F}_{q^{i}}\right)$ modulo $p^{l}$ then

$$
\# X\left(\mathbb{F}_{q^{i}}\right) \equiv \sum_{\left(z_{1}, \ldots, z_{N}\right) \in \mu_{q^{i}-1}^{N}} \Phi\left(z_{1}, \ldots, z_{N}\right) \quad\left(\bmod p^{l}\right) .
$$

(ii) One has

$$
\sum_{z \in \mu_{q^{i}-1}} z^{e}=0
$$

unless $e$ is a multiple of $q^{i}-1$. Therefore, the right-hand side of $(3)$ is $\left(q^{i}-1\right)^{N}$ times the sum of the coefficients of $\Phi$ at the monomials of the form $\left(T_{1}^{e_{1}} \ldots T_{N}^{e_{N}}\right)^{q^{i}-1}$, for $e_{1}, \ldots, e_{N} \in \mathbb{Z}_{\geqslant 0}$.

(iii) For $l=1$, these facts have been known for a long time; cf. [3, $\S 1.1$, Theorem 4].

(iv) One might want to take the step from $l=1$ to a larger value in a more naive manner as follows. For $\left(\pi\left(z_{1}\right), \ldots, \pi\left(z_{N}\right)\right) \notin X\left(\mathbb{F}_{q^{i}}\right)$, one has $N_{\mathbb{Z}_{q^{i}} / \mathbb{Z}_{q}} F^{a(q-1)}\left(z_{1}, \ldots, z_{N}\right)=1+s p$, for some unknown $s \in \mathbb{Z}_{q^{i}}$. This yields

$$
\begin{aligned}
N_{\mathbb{Z}_{q^{i}} / \mathbb{Z}_{q}} F^{2 a(q-1)}\left(z_{1}, \ldots, z_{N}\right)= & 1+2 s p+s^{2} p^{2}, \\
N_{\mathbb{Z}_{q^{i}} / \mathbb{Z}_{q}} F^{3 a(q-1)}\left(z_{1}, \ldots, z_{N}\right)= & 1+3 s p+3 s^{2} p^{2}+s^{3} p^{3}, \\
\vdots & \\
N_{\mathbb{Z}_{q^{i}} / \mathbb{Z}_{q}} F^{l a(q-1)}\left(z_{1}, \ldots, z_{N}\right)= & 1+l s p+\left(\begin{array}{c}
l \\
2
\end{array}\right) s^{2} p^{2}+\left(\begin{array}{l}
l \\
3
\end{array}\right) s^{3} p^{3} \\
& +\ldots+\left(\begin{array}{c}
l \\
l-1
\end{array}\right) s^{l-1} p^{l-1}+\left(\begin{array}{l}
l \\
l
\end{array}\right) s^{l} p^{l} .
\end{aligned}
$$

There is a linear combination of these expressions that eliminates all terms containing $p, p^{2}, \ldots, p^{l-1}$. Namely, one needs to take as the coefficient vector the first row of the Pascal-like matrix

$$
\left(\begin{array}{ccccc}
1 & 1 & 0 & \ldots & 0 \\
1 & 2 & 1 & \ldots & 0 \\
\vdots & \vdots & \vdots & \ddots & \vdots \\
1 & l-1 & \left(\begin{array}{c}
l-1 \\
2
\end{array}\right) & \cdots & \left(\begin{array}{c}
l-1 \\
l-1
\end{array}\right) \\
1 & l & \left(\begin{array}{l}
l \\
2
\end{array}\right) & \ldots & \left(\begin{array}{c}
l \\
l-1
\end{array}\right)
\end{array}\right)^{-1}
$$


However, well-known relations between binomial coefficients reveal that the first row of this matrix is exactly $\left(l,-\left(\begin{array}{l}l \\ 2\end{array}\right),\left(\begin{array}{l}l \\ 3\end{array}\right), \ldots,(-1)^{l+1}\left(\begin{array}{l}l \\ l\end{array}\right)\right)$, in agreement with Proposition 3.3.

\section{Linear operators}

Notation 3.5. (i) We consider the polynomial ring $\mathbb{Z}_{q}\left[T_{1}, \ldots, T_{N}\right]$ as a free $\mathbb{Z}_{q}$-module and equip it with the symmetric bilinear form $\langle.,$.$\rangle , given by$

$$
\left\langle\sum_{i_{1}, \ldots, i_{N}} a_{i_{1}, \ldots, i_{N}} T_{1}^{i_{1}} \ldots T_{N}^{i_{N}}, \sum_{i_{1}, \ldots, i_{N}} b_{i_{1}, \ldots, i_{N}} T_{1}^{i_{1}} \ldots T_{N}^{i_{N}}\right\rangle:=\sum_{i_{1}, \ldots, i_{N}} a_{i_{1}, \ldots, i_{N}} b_{i_{1}, \ldots, i_{N}} .
$$

Then the monomials form an orthonormal basis. Furthermore, the bilinear form $\langle.,$.$\rangle yields$ a norm $\|\cdot\|$ on $\mathbb{Z}_{q}\left[T_{1}, \ldots, T_{N}\right]$ such that $\|g\|:=\left[\|\langle g, g\rangle\|_{p}\right]^{1 / 2}$, for $\|\cdot\|_{p}$ the usual normalised $p$-adic valuation. The completion $\mathbb{Z}_{q}\left\{\left\{T_{1}, \ldots, T_{N}\right\}\right\}$ is a $\mathbb{Z}_{q}$-Hilbert space.

(ii) There is the bounded linear operator $\varphi: \mathbb{Z}_{q}\left\{\left\{T_{1}, \ldots, T_{N}\right\}\right\} \rightarrow \mathbb{Z}_{q}\left\{\left\{T_{1}, \ldots, T_{N}\right\}\right\}$, provided by taking the quasi power $h \mapsto h^{(q)}$. Its adjoint

$$
\kappa: \mathbb{Z}_{q}\left\{\left\{T_{1}, \ldots, T_{N}\right\}\right\} \longrightarrow \mathbb{Z}_{q}\left\{\left\{T_{1}, \ldots, T_{N}\right\}\right\}
$$

maps the monomial $\left(T_{1}^{e_{1}} \ldots T_{N}^{e_{N}}\right)^{q}$ to $T_{1}^{e_{1}} \ldots T_{N}^{e_{N}}$ and all monomials not being of this particular type to zero. In particular, $\kappa$ is a retraction for $\varphi$. That is, one has $\kappa \circ \varphi=$ id.

On the other hand, for $i \in \mathbb{N}$, the composition

$$
\varphi^{i} \circ \kappa^{i}: \mathbb{Z}_{q}\left\{\left\{T_{1}, \ldots, T_{N}\right\}\right\} \rightarrow \mathbb{Z}_{q}\left\{\left\{T_{1}, \ldots, T_{N}\right\}\right\}
$$

is the orthogonal projection to the sub- $\mathbb{Z}_{q}$-Hilbert space spanned by all monomials of the type $\left(T_{1}^{e_{1}} \ldots T_{N}^{e_{N}}\right)^{q^{i}}$.

(iii) For every polynomial $g \in \mathbb{Z}_{q}\left[T_{1}, \ldots, T_{N}\right]$, the multiplication map

$$
m_{g}: \mathbb{Z}_{q}\left\{\left\{T_{1}, \ldots, T_{N}\right\}\right\} \longrightarrow \mathbb{Z}_{q}\left\{\left\{T_{1}, \ldots, T_{N}\right\}\right\}, \quad h \mapsto g h,
$$

is a bounded linear operator.

FACT 3.6. Let $g \in \mathbb{Z}_{q}\left[T_{1}, \ldots, T_{N}\right]$ and $i \in \mathbb{N}$ be arbitrary. Then

$$
\kappa^{i} \circ m_{g^{\left(q^{i}\right)}} \circ \varphi^{i}=m_{g} .
$$

Definition 3.7 (Linear operator associated with a polynomial). Given a positive integer $i$ and a polynomial $g \in \mathbb{Z}_{q}\left[T_{1}, \ldots, T_{N}\right]$, we consider the linear operator

$$
\mathrm{M}_{g, i}:=\kappa^{i} \circ m_{g} .
$$

This operator is of interest because of the two results below.

Lemma 3.8. For every positive integer $i$ and every polynomial $g \in \mathbb{Z}_{q}\left[T_{0}, \ldots, T_{N}\right]$, one has

$$
\mathrm{M}_{\mathrm{N}_{\mathbb{Z}_{q^{i}} / \mathbb{Z}_{q}} g, i}=\left(\mathrm{M}_{g, 1}\right)^{i} .
$$

Proof. We will show this inductively. The assertion is clearly true for $i=1$. For the inductive step, let us assume that equation (4) is true for a certain value of $i$. Then, by 3.1(iii), we have $\mathrm{N}_{\mathbb{Z}_{q^{i+1}} / \mathbb{Z}_{q}} g=g^{\left(q^{i}\right)} \cdot \mathrm{N}_{\mathbb{Z}_{q^{i}} / \mathbb{Z}_{q}} g$. Hence, Definition 3.7 shows that

$$
\begin{aligned}
\mathrm{M}_{\mathrm{N}_{\mathbb{Z}_{q^{i+1}} / \mathbb{Z}_{q}} g, i+1} & =\kappa^{i+1} \circ m_{\mathrm{N}_{\mathbb{Z}_{q^{i+1}} / \mathbb{Z}_{q}} g} \\
& =\kappa^{i+1} \circ m_{g^{\left(q^{i}\right)}} \circ m_{\mathrm{N}_{\mathbb{Z}_{q^{i}} / \mathbb{Z}_{q}} g} \\
& =\kappa^{i+1} \circ m_{g^{\left(q^{i}\right)}} \circ \varphi^{i} \circ \kappa^{i} \circ m_{\mathrm{N}_{\mathbb{Z}_{q^{i}} / \mathbb{Z}_{q}} g} .
\end{aligned}
$$


Observe that the projection $\varphi^{i}$ o ${ }^{i}$ has no effect here. Indeed, it is followed by multiplication with a polynomial all of whose monomials have exponents only divisible by $q^{i}$, and the retraction $\kappa^{i}$. Thus, according to Fact 3.6, Definition 3.7 and the induction hypothesis,

$$
\begin{aligned}
\mathrm{M}_{\mathrm{N}_{\mathbb{Z}_{q} i+1} / \mathbb{Z}_{q} g, i+1} & =\kappa \circ m_{g} \circ \mathrm{M}_{\mathrm{N}_{\mathbb{Z}_{q} / \mathbb{Z}_{q}} g, i} \\
& =\mathrm{M}_{g, 1} \circ\left(\mathrm{M}_{g, 1}\right)^{i} \\
& =\left(\mathrm{M}_{g, 1}\right)^{i+1}
\end{aligned}
$$

as required.

Proposition 3.9. Let $i$ be a positive integer and $g \in \mathbb{Z}_{q}\left[T_{0}, \ldots, T_{N}\right]$ be any polynomial.

(a) Then the linear operator $\mathrm{M}_{g, i}$ is trace class.

(b) Its trace $\operatorname{tr} \mathrm{M}_{g, i}$ is equal to the sum of all coefficients of $g$ at the monomials of the form $\left(T_{1}^{e_{1}} \ldots T_{N}^{e_{N}}\right)^{q^{i}-1}$.

Proof. We will only prove (b), as this immediately implies (a). By definition,

$$
\operatorname{tr} \mathrm{M}_{g, i}=\sum_{k \in K}\left\langle\mathrm{M}_{g, i}\left(v_{k}\right), v_{k}\right\rangle
$$

for $\left(v_{k}\right)_{k \in K}$ any complete orthonormal system; in particular, for the system of all monomials.

For the monomial $T_{1}^{e_{1}} \ldots T_{N}^{e_{N}}$, the pairing $\left\langle\mathrm{M}_{g, i}\left(T_{1}^{e_{1}} \ldots T_{N}^{e_{N}}\right), T_{1}^{e_{1}} \ldots T_{N}^{e_{N}}\right\rangle$ yields the coefficient of $\mathrm{M}_{g, i}\left(T_{1}^{e_{1}} \ldots T_{N}^{e_{N}}\right)=\kappa^{i}\left(g T_{1}^{e_{1}} \ldots T_{N}^{e_{N}}\right)$ at the monomial $T_{1}^{e_{1}} \ldots T_{N}^{e_{N}}$. This is nothing but the coefficient of $g T_{1}^{e_{1}} \ldots T_{N}^{e_{N}}$ at $\left(T_{1}^{e_{1}} \ldots T_{N}^{e_{N}}\right)^{q^{i}}$ and, hence, the coefficient of $g$ at $\left(T_{1}^{e_{1}} \ldots T_{N}^{e_{N}}\right)^{q^{i}-1}$. The assertion follows.

Remark 3.10. For general information on $p$-adic Hilbert spaces, linear operators and the concept of the trace, the interested reader might compare Chapters 2 and 3 of Diagana's textbook [12].

\section{An algorithm}

Algorithm 3.11. Given a projective hypersurface $X=V(f) \subset \mathbf{P}_{\mathbb{F}_{q}}^{N}$ and a positive integer $n$, this algorithm computes the numbers of points $\# X\left(\mathbb{F}_{q}\right), \# X\left(\mathbb{F}_{q^{2}}\right), \ldots, \# X\left(\mathbb{F}_{q^{n}}\right)$.

(i) Choose a lift $\widetilde{F} \in \mathbb{Z}_{q}\left[T_{0}, \ldots, T_{N}\right]$ of $f$. Decompose $X$ into $X^{c}:=\left[V\left(T_{0}\right) \cup \ldots \cup V\left(T_{N}\right)\right] \cap X$ and $X^{0}:=X \backslash X^{c}$. For the lower-dimensional closed subscheme $X^{c}$, either use naive pointcounting methods or apply the method recursively. For $X^{0}$, do the following.

(ii) Use elementary arguments or the Weil conjectures or both to find estimates for $\# X^{0}\left(\mathbb{F}_{q}\right)$, $\# X^{0}\left(\mathbb{F}_{q^{2}}\right), \ldots, \# X^{0}\left(\mathbb{F}_{q^{n}}\right)$. From these, deduce a $p$-adic precision $l$ that suffices to determine these numbers exactly. Finally, put $a:=\lceil l /(q-1)\rceil$.

(iii) Define $F \in \mathbb{Z}_{q}\left[T_{1}, \ldots, T_{N}\right]$ by putting $F\left(T_{1}, \ldots, T_{N}\right):=\widetilde{F}\left(1, T_{1}, \ldots, T_{N}\right)$.

(iv) Find matrix representations of the operators $\mathrm{M}_{F^{a(q-1)}, 1}, \mathrm{M}_{F^{2 a(q-1)}, 1}, \ldots, \mathrm{M}_{F^{l a(q-1)}, 1}$. Work on finite-dimensional subspaces that are large enough to get the traces right. For a polynomial of total degree $d$, as a basis one may use all monomials of total degree less than or equal to $\lceil d /(q-1)\rceil$.

(v) For $i:=1$ to $n$, do the following. Calculate the matrix powers $\mathrm{M}_{F^{a(q-1)}, 1}^{i}$, $\mathrm{M}_{F^{2 a(q-1)}, 1}^{i}, \ldots, \mathrm{M}_{F^{l a(q-1)}, 1}^{i}$ and eventually their traces $\operatorname{tr} \mathrm{M}_{F^{k a(q-1)}, 1}^{i}$. Then

$$
\# X^{0}\left(\mathbb{F}_{q^{i}}\right) \equiv\left(q^{i}-1\right)^{N}\left[1+\sum_{k=1}^{l}(-1)^{k}\left(\begin{array}{l}
l \\
k
\end{array}\right) \operatorname{tr} \mathrm{M}_{F^{k a(q-1)}, 1}^{i}\right] \quad\left(\bmod p^{l}\right) .
$$


Determine the exact value of $\# X^{0}\left(\mathbb{F}_{q^{i}}\right)$ from this congruence and the estimate established in step (ii). Add $\# X^{c}\left(\mathbb{F}_{q^{i}}\right)$ and output the integer found.

REMARKs 3.12. (i) Formula (5) is essentially the same as the 'trace formula' in $[\mathbf{2 6}$, Theorem 3.1].

(ii) (Cohomological interpretation) The matrix $\mathrm{M}_{F^{q-1}, 1}$ appears for the first time in $[\mathbf{1 3}, \S 2]$ and later in [33, Corollaries 1 and 2], where it is shown to be the natural generalisation of the classical Hasse-Witt matrix [27]. In other words, it represents the Frobenius homomorphism on the middle coherent cohomology $H^{N-1}\left(X, \mathscr{O}_{X}\right)$. The characteristic polynomial of $\mathrm{M}_{F^{q-1}, 1}$ is hence congruent modulo $p$ to the Weil polynomial $\chi_{N-1}[\mathbf{1 1}$, Exp.XXII, Théorème 3.1] Unfortunately, the algorithm above does not provide a canonical lift representing the crystalline Frobenius, but only successive $p$-adic approximations of the proper eigenvalues, so that (5) finally holds due to many cancellations.

\section{A variation adapted to double covers}

The variation

Notation 4.1. (i) We assume in this section that $\mathbb{F}_{q}$ is a finite field of characteristic $p \neq 2$.

(ii) Furthermore, let $X$ be a double cover of an affine torus $\mathbb{G}_{m, \mathbb{F}_{q}}^{N}$, given by an equation of the form $w^{2}=f$ for a polynomial $f \in \mathbb{F}_{q}\left[T_{1}, \ldots, T_{N}\right]$. Our interest stems from double covers of projective space of $\mathbf{P}_{\mathbb{F}_{q}}^{N}$, but, as before, going from projective space to an affine torus is not a serious modification. One may at the very end cover $\mathbf{P}_{\mathbb{F}_{q}}^{N}$ by affine tori.

(iii) We choose a lift $F \in \mathbb{Z}_{q}\left[T_{1}, \ldots, T_{N}\right]$ of $f$.

(iv) For a positive integer $l$ and $k=1, \ldots, l$, let us put

$$
A_{k}^{(l)}:=\frac{(2 l) !}{2^{2 l-1} l !(l-1) !} \cdot \frac{(-1)^{k+1}}{2 k-1}\left(\begin{array}{l}
l-1 \\
k-1
\end{array}\right) .
$$

Lemma 4.2. Let $l>0$ and $b$ be integers.

(a) Then the Pascal-like matrix

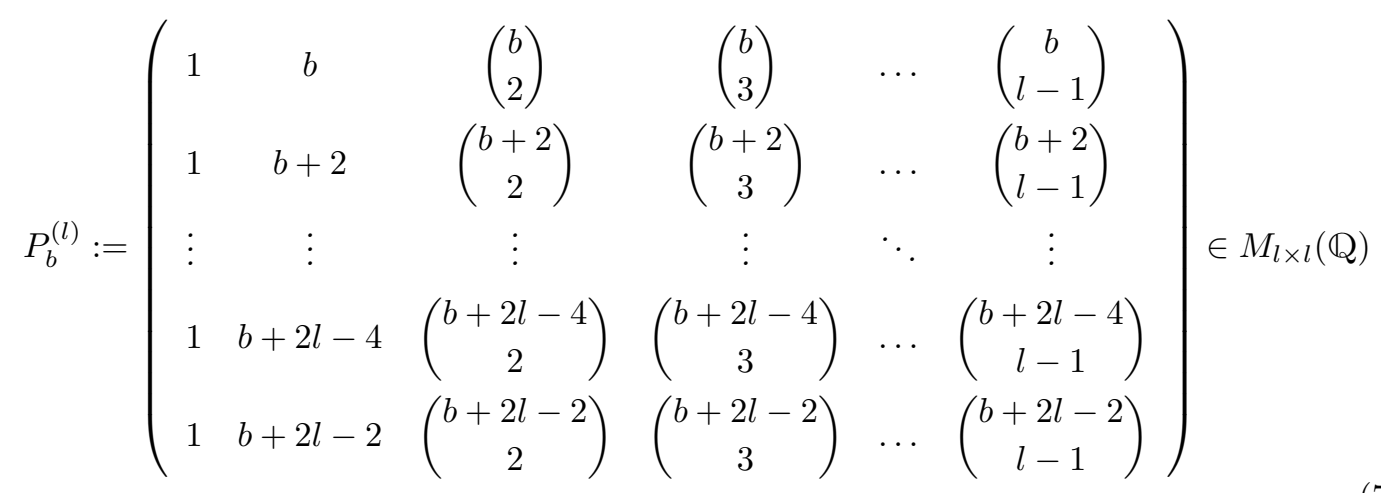

is invertible.

(b) All coefficients of its inverse $\left(P_{b}^{(l)}\right)^{-1}$ are $p$-adic integers.

(c) The first row of $\left(P_{1}^{(l)}\right)^{-1}$ is $\left(A_{1}^{(l)}, A_{2}^{(l)}, \ldots, A_{l}^{(l)}\right)$.

Proof. (a) and (b) Column transformations convert $P_{b}^{(l)}$ essentially into a Vandermonde matrix. This shows 


$$
\operatorname{det} P_{b}^{(l)}=\frac{(2 l-2) ! !(2 l-4) ! ! \ldots 2 ! !}{1 ! 2 ! \ldots(l-1) !}=2^{(l-1)+(l-2)+\ldots+1}=2^{\left(\begin{array}{l}
l \\
2
\end{array}\right)} \neq 0
$$

which immediately implies (a). Assertion (b) follows from this together with Cramer's rule.

(c) The assertion is that the linear combination with coefficients $\left(A_{1}^{(l)}, A_{2}^{(l)}, \ldots, A_{l}^{(l)}\right)$ of the rows of the matrix $P_{1}^{(l)}$ yields $(1,0, \ldots, 0)$. Here, the occurrence of the zeros is a consequence of the standard relation $\sum_{k=1}^{l}(-1)^{k}\left(\begin{array}{l}l-1 \\ k-1\end{array}\right) k^{e}=0$ among binomial coefficients, for $e=0, \ldots, l-2$. It is a trivial, but tedious exercise to adjust the constant factor.

Definition 4.3. We call a function $\Phi: \mu_{q^{i}-1}^{N} \rightarrow \mathbb{Z}_{q}$ an Eulerian function for $X\left(\mathbb{F}_{q^{i}}\right)$ modulo $p^{l}$ if, for every $\left(z_{1}, \ldots, z_{N}\right) \in \mu_{q^{i}-1}^{N}$,

$$
\begin{aligned}
& \Phi\left(z_{1}, \ldots, z_{N}\right) \equiv 1 \quad\left(\bmod p^{l}\right) \quad \text { if } f\left(\pi\left(z_{1}\right), \ldots, \pi\left(z_{N}\right)\right) \text { is a square in } \mathbb{F}_{q^{i}}^{*} \\
& \Phi\left(z_{1}, \ldots, z_{N}\right) \equiv-1 \quad\left(\bmod p^{l}\right) \quad \text { if } f\left(\pi\left(z_{1}\right), \ldots, \pi\left(z_{N}\right)\right) \text { is a non-square in } \mathbb{F}_{q^{i}}^{*},
\end{aligned}
$$

and

$$
\Phi\left(z_{1}, \ldots, z_{N}\right) \equiv 0 \quad\left(\bmod p^{l}\right) \quad \text { if } f\left(\pi\left(z_{1}\right), \ldots, \pi\left(z_{N}\right)\right)=0
$$

TheOrem 4.4. Suppose that $p$ is an odd prime and that $b$ is odd such that $b(q-1) / 2 \geqslant l$. Put $A_{b, k}^{(l)}:=\left(\left(P_{b}^{(l)}\right)^{-1}\right)_{1, k}$. Then

$$
\Phi:=\sum_{k=1}^{l} A_{b, k}^{(l)} \cdot N_{\mathbb{Z}_{q^{i}} / \mathbb{Z}_{q}} F^{(b+2 k-2)(q-1) / 2}
$$

is an Eulerian function for $X\left(\mathbb{F}_{q^{i}}\right)$ modulo $p^{l}$.

Proof. Recall that each of the coefficients $A_{b, 1}^{(l)}:=\left(\left(P_{b}^{(l)}\right)^{-1}\right)_{1,1}, \ldots, A_{b, l}^{(l)}:=\left(\left(P_{b}^{(l)}\right)^{-1}\right)_{1, l}$ is a $p$-adic integer. Furthermore, one has that $f\left(\pi\left(z_{1}\right), \ldots, \pi\left(z_{N}\right)\right)=0 \in \mathbb{F}_{q^{i}}$ if and only if $\mathrm{N}_{\mathbb{F}_{q^{i}} / \mathbb{F}_{q}}\left(f\left(\pi\left(z_{1}\right), \ldots, \pi\left(z_{N}\right)\right)\right)=0 \in \mathbb{F}_{q}$, which, in turn, is equivalent to $N_{\mathbb{Z}_{q^{i}} / \mathbb{Z}_{q}} F\left(z_{1}, \ldots, z_{N}\right)$ being a non-unit in $\mathbb{Z}_{q}$. If this is true then $N_{\mathbb{Z}_{q} / \mathbb{Z}_{q}} F^{(b+2 k-2)(q-1) / 2}\left(z_{1}, \ldots, z_{N}\right)$ is of $p$-adic valuation at least $(b+2 k-2)(q-1) / 2 \geqslant b(q-1) / 2 \geqslant l$. Consequently,

$$
\sum_{k=1}^{l} A_{b, k}^{(l)} \cdot N_{\mathbb{Z}_{q^{i}} / \mathbb{Z}_{q}} F^{(b+2 k-2)(q-1) / 2} \equiv 0 \quad\left(\bmod p^{l}\right) .
$$

Otherwise, $f\left(\pi\left(z_{1}\right), \ldots, \pi\left(z_{N}\right)\right) \in\left(\mathbb{F}_{q^{i}}^{*}\right)^{2}$ precisely when $\mathrm{N}_{\mathbb{F}_{q^{i}} / \mathbb{F}_{q}}\left(f\left(\pi\left(z_{1}\right), \ldots, \pi\left(z_{N}\right)\right)\right) \in \mathbb{F}_{q}^{*}$ is a square. Then

$$
\mathrm{N}_{\mathbb{F}_{q^{i}} / \mathbb{F}_{q}}\left(f^{(q-1) / 2}\left(\pi\left(z_{1}\right), \ldots, \pi\left(z_{N}\right)\right)\right)=1 \in \mathbb{F}_{q},
$$

while the same expression is equal to $(-1)$ in the case of a non-square. By construction, this means $N_{\mathbb{Z}_{q^{i}} / \mathbb{Z}_{q}} F^{(q-1) / 2}\left(z_{1}, \ldots, z_{N}\right) \equiv \pm 1(\bmod p)$, respectively.

Write $N_{\mathbb{Z}_{q^{i}} / \mathbb{Z}_{q}} F^{(q-1) / 2}\left(z_{1}, \ldots, z_{N}\right)= \pm(1+s p)$ for some unknown $s \in \mathbb{Z}_{q^{i}}$. This yields

$$
\begin{aligned}
& N_{\mathbb{Z}_{q^{i}} / \mathbb{Z}_{q}} F^{3(q-1) / 2}\left(z_{1}, \ldots, z_{N}\right)= \pm\left(1+3 s p+3 s^{2} p^{2}+s^{3} p^{3}\right), \\
& N_{\mathbb{Z}_{q^{i}} / \mathbb{Z}_{q}} F^{5(q-1) / 2}\left(z_{1}, \ldots, z_{N}\right)= \pm\left(1+5 s p+10 s^{2} p^{2}+10 s^{3} p^{3}+5 s^{4} p^{4}+s^{5} p^{5}\right), \\
& N_{\mathbb{Z}_{q^{i}} / \mathbb{Z}_{q}} F^{(b+2 l-2)(q-1) / 2}\left(z_{1}, \ldots, z_{N}\right)= \pm\left(1+(b+2 l-2) s p+\left(\begin{array}{c}
b+2 l-2 \\
2
\end{array}\right) s^{2} p^{2}\right. \\
& \left.+\left(\begin{array}{c}
b+2 l-2 \\
3
\end{array}\right) s^{3} p^{3}+\ldots+\left(\begin{array}{c}
b+2 l-2 \\
l-1
\end{array}\right) s^{l-1} p^{l-1}+\ldots\right) .
\end{aligned}
$$


Lemma 4.2(a) and (b) now show

$$
\sum_{k=1}^{l} A_{b, k}^{(l)} \cdot N_{\mathbb{Z}_{q^{i}} / \mathbb{Z}_{q}} F^{(b+2 k-2)(q-1) / 2}\left(z_{1}, \ldots, z_{N}\right) \equiv \pm 1\left(\bmod p^{l}\right),
$$

as required.

REMARK 4.5. According to the definition, if $\Phi$ is an Eulerian function for $X\left(\mathbb{F}_{q^{i}}\right)$ modulo $p^{l}$ then

$$
\# X\left(\mathbb{F}_{q^{i}}\right) \equiv\left(q^{i}-1\right)^{N}+\sum_{\left(z_{1}, \ldots, z_{N}\right) \in \mu_{q^{i}-1}^{N}} \Phi\left(z_{1}, \ldots, z_{N}\right) \quad\left(\bmod p^{l}\right) .
$$

This leads to the algorithm below.

Algorithm 4.6. Given a polynomial $f \in \mathbb{F}_{q}\left[T_{0}, \ldots, T_{N}\right]$ and a positive integer $n$, this algorithm computes the numbers of points $\# X\left(\mathbb{F}_{q}\right), \# X\left(\mathbb{F}_{q^{2}}\right), \ldots, \# X\left(\mathbb{F}_{q^{n}}\right)$ on the double cover of $\mathbf{P}_{\mathbb{F}_{q}}^{N}$, given by $w^{2}=f$.

(i) Choose a lift $\widetilde{F} \in \mathbb{Z}_{q}\left[T_{0}, \ldots, T_{N}\right]$ of $f$. Decompose $\mathbf{P}_{\mathbb{F}_{q}}^{N}$ into $P^{c}:=V\left(T_{0}\right) \cup \ldots \cup V\left(T_{N}\right)$ and $P^{0}:=\mathbf{P}_{\mathbb{F}_{q}}^{N} \backslash P^{c}$. Then $P^{0} \cong \mathbb{G}_{m, \mathbb{F}_{q}}^{N}$. For the restriction $X^{c}$ of the double cover to the lower-dimensional closed subscheme $P^{c}$, either use naive point-counting methods or apply the method recursively. For $X^{0}$, the restriction to $P^{0}$, do the following.

(ii) Use elementary arguments or the Weil conjectures or both to find estimates for $\# X^{0}\left(\mathbb{F}_{q}\right)$, $\# X^{0}\left(\mathbb{F}_{q^{2}}\right), \ldots, \# X^{0}\left(\mathbb{F}_{q^{n}}\right)$. From these, deduce a $p$-adic precision $l$ that suffices to determine these numbers exactly. Finally, put $b:=\lfloor 2 l /(q-1)\rfloor$.

(iii) If $b>1$ then determine the coefficient vector $\left(A_{b, 1}^{(l)}, \ldots, A_{b, l}^{(l)}\right)$ by inverting the matrix $P_{b}^{(l)}$ from (7). Otherwise, calculate first

$$
C_{l}:=\frac{(2 l) !}{2^{2 l-1} l !(l-1) !}
$$

and then the coefficients

$$
A_{k}^{(l)}:=C_{l} \cdot \frac{(-1)^{k+1}}{2 k-1}\left(\begin{array}{l}
l-1 \\
k-1
\end{array}\right)
$$

Finally, set $\left(A_{1,1}^{(l)}, \ldots, A_{1, l}^{(l)}\right):=\left(A_{1}^{(l)}, \ldots, A_{l}^{(l)}\right)$.

(iv) Define $F \in \mathbb{Z}_{q}\left[T_{1}, \ldots, T_{N}\right]$ by putting $F\left(T_{1}, \ldots, T_{N}\right):=\widetilde{F}\left(1, T_{1}, \ldots, T_{N}\right)$.

(v) Find matrix representations of the operators $\mathrm{M}_{F^{(q-1) / 2}, 1}, \mathrm{M}_{F^{3(q-1 / 2)}, 1}, \ldots$, $\mathrm{M}_{F^{(b+2 l-2)(q-1 / 2), 1}}$. Work on finite-dimensional subspaces that are large enough to get the traces right. For a polynomial of total degree $d$, as a basis one may use all monomials of total degree less than or equal to $\lceil d /(q-1)\rceil$.

(vi) For $i:=1$ to $n$, do the following.

Calculate the matrix powers $\mathrm{M}_{F^{(q-1) / 2}, 1}^{i}, \mathrm{M}_{F^{3(q-1) / 2}, 1}^{i}, \ldots, \mathrm{M}_{F^{(b+2 l-2)(q-1) / 2,1}}^{i}$ and eventually their traces $\operatorname{tr} \mathrm{M}_{F^{(b+2 k-2)(q-1) / 2}, 1}^{i}$. Then

$$
\# X^{0}\left(\mathbb{F}_{q^{i}}\right) \equiv\left(q^{i}-1\right)^{N}\left[1+\sum_{k=1}^{l} A_{b, k}^{(l)} \cdot \operatorname{tr} \mathrm{M}_{F^{(b+2 k-2)(q-1) / 2}, 1}^{i}\right] \quad\left(\bmod p^{l}\right) .
$$

Determine the exact value of $\# X^{0}\left(\mathbb{F}_{q^{i}}\right)$ from this congruence and the estimate established in step (ii). Add \# $X^{c}\left(\mathbb{F}_{q^{i}}\right)$ and output the integer found.

Remarks 4.7. (i) (Complexity) The algorithm contains two time-consuming operations: the first is the computation of the powers of $F$, and the second is the multiplication of matrices. We tested our implementation in magma on one core of an Intel i5-4690 processor running at $3.5 \mathrm{GHz}$. 
For a K3 surface of degree 2, in order to compute the whole Weil polynomial, the algorithm has to work with a $p$-adic precision of at least 11 digits. For this, it has to compute $F^{k(p-1) / 2}$ for all odd integers $1 \leqslant k \leqslant 21$. This results in matrices of size up to $2080 \times 2080$ having entries in $\mathbb{Z} / p^{11} \mathbb{Z}$.

Table 1 lists the time used for a randomly chosen surface in a test modulo four representative primes of good reduction. The computation required about $13 \mathrm{~GB}$ of memory.

The figures show that the time for the computation of the powers of $F$ is approximately cubic in $p$. This is explained by the fact that the number of terms of the final result is quadratic in $p$, while the number of multiplications necessary to approach it is linear in $p$. The time for the matrix operations is almost constant as the size of the matrices is constant. The variation of the time is explained completely by the change of the ring the matrix entries are taken from.

In theory, there exists an algorithm that is quadratic in $p$. This is, however, not yet implemented in magma and, reportedly, it is not easy to implement.

(ii) We incorporated the variable $b$ only for completeness. In practice, the algorithm is of interest particularly when $q$ is not very small. Then $q-1 \geqslant 2 l$ and $b=1$. The same applies to the variable $a$ in Algorithm 3.11.

(iii) One might as well determine the coefficient vector $\left(A_{b, 1}^{(l)}, \ldots, A_{b, l}^{(l)}\right)$ by a matrix inversion, also when $b=1$. This step is in fact not time-critical. Nevertheless, we find it interesting that it is possible to give an explicit formula.

REMARK 4.8 (The moving simplex idea). The idea behind most of the operator calculus above is that only very few of the coefficients of the powers of $F$ are actually needed. The following is an extreme case. Assume that a K3 surface $X$ is given as a double cover of $\mathbf{P}_{\mathbb{Q}}^{2}$, by the equation $W^{2}=F\left(T_{0}, T_{1}, T_{2}\right)$ for $F$ a homogeneous polynomial of degree 6 . Moreover, one only wants to decide for which primes $p$ up to a certain bound $B$ the reduction $X_{p}$ is ordinary. This means that only $\left(\# X_{p}\left(\mathbb{F}_{p}\right) \bmod p\right)$ is required. Thus, for each prime, just a single coefficient is to be computed, that of $F^{(p-1) / 2}$ at $T_{0}^{p-1} T_{1}^{p-1} T_{2}^{p-1}$.

This might be done as follows. Consider just a small triangle of coefficients of $F^{e}$, those at $T_{0}^{a_{0}} T_{1}^{a_{1}} T_{2}^{a_{2}}$ such that $\left|a_{i}-\left(a_{0}+a_{1}+a_{2}\right) / 3\right| \leqslant c_{i}$ for $i=0,1,2$ and some constants $c_{0}$, $c_{1}$, and $c_{2}$. These triangles may be calculated inductively for $e=1, \ldots,(B-1) / 2$. For $F$ homogeneous of degree $d$, any choice such that $c_{0}+c_{1}+c_{2}:=2(d-1)$ should work.

Indeed, $F$ is given and therefore $d F / d T_{0}, d F / d T_{1}$, and $d F / d T_{2}$ are known. When the triangle for $F^{e}$ has been established, computing $F^{e+1}$ naively would mean the loss of some of the coefficients. But the coefficients of $F^{e+1}$ have massive linear relations. A single coefficient of $F^{e+1}$ appears as a linear combination of the coefficients of $F^{e}$, when evaluating the formula $F^{e+1}=F \cdot F^{e}$. It appears as well in $\left(d F^{e+1} / d T_{0}\right)=(e+1) F^{e}\left(d F / d T_{0}\right)$ and the analogous formulae involving $T_{1}$ and $T_{2}$. Hence, each hypothetical coefficient yields three linear relations. One may thus reconstruct some neighbouring coefficients using the linear relations and therefore keep the size of the triangle constant under iteration. This approach stems from $[\mathbf{2 6}, \S 4.1]$ and is implemented in the function NonOrdinaryPrimes.

TABLE 1. Time (in s) for the steps of the algorithm.

\begin{tabular}{rccc}
\hline \multicolumn{1}{c}{$p$} & Powers of $F$ & Matrix build & Matrix operations \\
\hline 31 & 12.65 & 21.05 & 55.36 \\
61 & 76.91 & 21.63 & 71.00 \\
97 & 236.92 & 22.30 & 73.53 \\
127 & 489.92 & 22.36 & 73.97 \\
\hline
\end{tabular}


On the other hand, WeilPolynomialOfDegree2K3Surface does not use the moving simplex idea, but the approach described above in this section. The point is that, for example for $p=127$, one has to compute $F^{63 \cdot 21}$, which results in a sum of 31517830 terms. Thereby, from the coefficients, about $14 \%$ are used as matrix entries. Thus, computing only the used coefficients of the powers of $F$ would speed up the algorithm only for considerably larger values of $p$.

REMARK 4.9. There are improvements to the moving simplex idea which we did not implement. The point is that instead of keeping track of the coefficients of $F^{e}$ on the entire simplex, one may sometimes, and probably often, get by with working only on a relatively small subset. For example, the idea has been worked out for smooth space quartics, in which case 64 coefficients suffice out of the 220 one would naively have. This is called the controlled reduction Abbott-Kedlaya-Roe algorithm [1, Algorithm 3.4.10]; cf. [7].

\section{Families of K3 surfaces that are highly likely to have real or complex multiplication}

5.1. Based on the Mumford-Tate conjecture, which was proven for K3 surfaces by Tankeev $[37,38]$, we established in $[20]$ certain arithmetic consequences of real or complex multiplication for K3 surfaces defined over $\mathbb{Q}$. Assume for simplicity that the endomorphism field $E$ is a quadratic number field. Then these consequences include the following.

(i) If $p$ is inert in $E$ then $\# X_{p}\left(\mathbb{F}_{p}\right) \equiv 1(\bmod p)$. That is, $X_{p}$ is non-ordinary.

(ii) For every prime $p$ of good reduction, the transcendental factor $\chi_{p}^{\operatorname{tr}}$ of the Weil polynomial of $X_{p}$ either splits over $E$ into two factors conjugate to each other or becomes a square under raising all its roots to the $f$ th power, for some $f>0$.

In recent years, we have systematically searched for K3 surfaces, the reductions of which show such an unusually regular behaviour. So far, we have the following conjectural list of suspicious surfaces.

Conjectures 5.2. Consider the following K3 surfaces and families of such:

$$
\begin{aligned}
V_{a}^{(2)}: W^{2}= & {\left[\left(\frac{1}{8} a^{2}-\frac{1}{2} a+\frac{1}{4}\right) T_{1}^{2}+\left(a^{2}-2 a+2\right) T_{1} T_{2}+\left(a^{2}-4 a+2\right) T_{2}^{2}\right] } \\
& \times\left[\left(\frac{1}{8} a^{2}+\frac{1}{2} a+\frac{1}{4}\right) T_{0}^{2}+\left(a^{2}+2 a+2\right) T_{0} T_{2}+\left(a^{2}+4 a+2\right) T_{2}^{2}\right] \\
& \times\left[2 T_{0}^{2}+\left(a^{2}+2\right) T_{0} T_{1}+a^{2} T_{1}^{2}\right] \\
V_{a}^{(5)}: W^{2}= & {\left[T_{1}^{2}+a T_{1} T_{2}+\left(\frac{5}{16} a^{2}+\frac{5}{4} a+\frac{5}{4}\right) T_{2}^{2}\right]\left[T_{0}^{2}+T_{0} T_{2}+\left(\frac{1}{320} a^{2}+\frac{1}{16} a+\frac{5}{16}\right) T_{2}^{2}\right] } \\
& \times\left[T_{0}^{2}+T_{0} T_{1}+\frac{1}{20} T_{1}^{2}\right] \\
V^{(13)}: W^{2}= & \left(25 T_{1}^{2}+26 T_{1} T_{2}+13 T_{2}^{2}\right)\left(T_{0}^{2}+2 T_{0} T_{2}+13 T_{2}^{2}\right)\left(9 T_{0}^{2}+26 T_{0} T_{1}+13 T_{1}^{2}\right), \\
V_{a, b}^{(2)}: W^{2}= & T_{0} T_{1} T_{2} f_{a, b} \quad \text { for } \\
f_{a, b}:= & a\left(-T_{0}^{2} T_{1}+T_{0}^{2} T_{2}+2 T_{0} T_{1}^{2}-3 T_{0} T_{1} T_{2}+T_{0} T_{2}^{2}+T_{1}^{3}-4 T_{1}^{2} T_{2}+5 T_{1} T_{2}^{2}-2 T_{2}^{3}\right) \\
& +b\left(T_{0}^{3}-2 T_{0}^{2} T_{1}-T_{0} T_{1}^{2}+3 T_{0} T_{1} T_{2}-T_{0} T_{2}^{2}+T_{1}^{2} T_{2}-T_{1} T_{2}^{2}\right), \\
V_{a, b}^{(3)}: W^{2}= & T_{0} T_{1} T_{2} f_{a, b} \quad \text { for } \\
f_{a, b}:= & \left(T_{0}^{3}-2 T_{0}^{2} T_{2}-T_{0} T_{1}^{2}-T_{0} T_{2}^{2}-2 T_{1}^{2} T_{2}+2 T_{2}^{3}\right) a^{2} \\
& +\left(6 T_{0}^{2} T_{1}+6 T_{0}^{2} T_{2}+6 T_{0} T_{1}^{2}+6 T_{1}^{2} T_{2}-6 T_{2}^{3}\right) a b \\
& +\left(-3 T_{0}^{2} T_{1}-6 T_{0}^{2} T_{2}+3 T_{1}^{3}-6 T_{1}^{2} T_{2}-3 T_{1} T_{2}^{2}+6 T_{2}^{3}\right) b^{2}, \\
V_{\mathbf{a}, \mathbf{b}}^{(-1)}: W^{2}= & T_{0} T_{1} T_{2}\left(T_{0}+T_{1}+T_{2}\right)\left(a_{1} T_{0}+a_{2} T_{1}+a_{3} T_{2}\right)\left(b_{1} T_{0}+b_{2} T_{1}+b_{3} T_{2}\right) \\
& \text { for } \mathbf{a}, \mathbf{b} \in \mathbb{C}^{3} \text { such that } a_{1} b_{3}+a_{2} b_{1}-2 a_{3} b_{1}=0 \quad \text { and } \\
& a_{1} b_{2}+a_{2} b_{3}-2 a_{3} b_{2}=0,
\end{aligned}
$$




$$
\begin{aligned}
V^{\left(-1, \mu_{7}\right)}: W^{2}= & T_{0} T_{1} T_{2}\left(7 T_{0}^{3}-7 T_{0}^{2} T_{1}+49 T_{0}^{2} T_{2}-21 T_{0} T_{1} T_{2}\right. \\
& \left.+98 T_{0} T_{2}^{2}+T_{1}^{3}-7 T_{1}^{2} T_{2}+49 T_{2}^{3}\right) \\
V^{\left(-1, \mu_{9}\right)}: W^{2}= & T_{0} T_{1} T_{2}\left(T_{0}^{3}-3 T_{0}^{2} T_{2}-3 T_{0} T_{1}^{2}-3 T_{0} T_{1} T_{2}+T_{1}^{3}+9 T_{1}^{2} T_{2}+6 T_{1} T_{2}^{2}+T_{2}^{3}\right), \\
V^{\left(-1, \mu_{19}\right)}: W^{2}= & T_{0} T_{1} T_{2}\left(49 T_{0}^{3}-304 T_{0}^{2} T_{1}+570 T_{0}^{2} T_{2}+361 T_{0} T_{1}^{2}-2793 T_{0} T_{1} T_{2}+2033 T_{0} T_{2}^{2}\right. \\
& \left.+361 T_{1}^{3}+2888 T_{1}^{2} T_{2}-5415 T_{1} T_{2}^{2}+2299 T_{2}^{3}\right) .
\end{aligned}
$$

(a) Then the generic fibre of each family, as well as each of the four individual surfaces, has geometric Picard rank 16.

(b) Moreover, the generic fibre of $V_{a}^{(2)}$ has real multiplication by $\mathbb{Q}(\sqrt{2})$, that of $V_{a}^{(5)} R M$ by $\mathbb{Q}(\sqrt{5})$, that of $V_{a, b}^{(2)} R M$ by $\mathbb{Q}(\sqrt{2})$, that of $V_{a, b}^{(3)} R M$ by $\mathbb{Q}(\sqrt{3})$, and that of $V_{\mathbf{a}, \mathbf{b}}^{(-1)}$ complex multiplication by $\mathbb{Q}(\sqrt{-1})$.

Finally, $V^{(13)}$ has $R M$ by $\mathbb{Q}(\sqrt{13}), V^{\left(-1, \mu_{7}\right)} C M$ by $\mathbb{Q}\left(\zeta_{28}+\zeta_{28}^{13}\right)=\mathbb{Q}\left(i, \zeta_{7}+\zeta_{7}^{-1}\right), V^{\left(-1, \mu_{9}\right)}$ $C M$ by $\mathbb{Q}\left(\zeta_{36}+\zeta_{36}^{17}\right)=\mathbb{Q}\left(i, \zeta_{9}+\zeta_{9}^{-1}\right)$, and $V^{\left(-1, \mu_{19}\right)} C M$ by $L(i)$, for $L \subset \mathbb{Q}\left(\mu_{19}\right)$ the unique cubic subfield.

REMARKS 5.3. (i) The equations given actually describe singular models of the K3 surfaces to be considered.

(ii) (Evidence) Thanks to Harvey's p-adic point-counting method, we can now give a lot more numerical evidence for our conjectures than before [20].

(iii) (Proven cases) The first three examples were already published in [20]. For the family $V_{a}^{(2)}$, both (a) and (b) were proven in [20, Theorem 6.6]. The same method applies to $V_{\mathbf{a}, \mathbf{b}}^{(-1)}$ and provides a proof also for this family. As far as only (a) is concerned, using van Luijk's method one shows in each case that the geometric Picard rank is 16 or 17, and 16 as soon as (b) is true.

(iv) In particular, for each family or surface, we explicitly know 16 divisors that are linearly independent in the Picard group. In neither case are all of them defined over $\mathbb{Q}$. For instance, for $V^{\left(-1, \mu_{7}\right)}, V^{\left(-1, \mu_{9}\right)}$ and $V^{\left(-1, \mu_{19}\right)}$, the fields of definition are $\mathbb{Q}\left(\zeta_{7}+\zeta_{7}^{-1}\right), \mathbb{Q}\left(\zeta_{9}+\zeta_{9}^{-1}\right)$ and the unique cubic subfield of $\mathbb{Q}\left(\mu_{19}\right)$, respectively.

On the other hand, for $V_{a}^{(2)}$, the field of definition of the known divisors is $\mathbb{Q}(\sqrt{2})$, while it is $\mathbb{Q}\left(\sqrt{5}, \sqrt{a^{2}-20 a-20}\right)$ for $V_{a}^{(5)}, \mathbb{Q}(\sqrt{13}, \sqrt{-3})$ for $V^{(13)}, \mathbb{Q}\left(\sqrt{2}, \sqrt{a}, \sqrt{b}, \sqrt{a^{2}-6 a b+b^{2}}\right)$ for $V_{a, b}^{(2)}$ and $\mathbb{Q}\left(\sqrt{3}, \sqrt{a^{4}-12 a^{3} b+30 a^{2} b^{2}-36 a b^{3}+9 b^{4}}, \sqrt{2 a^{2}-6 a b+6 b^{2}}\right)$ for $V_{a, b}^{(3)}$. We do not know whether the following is more than a coincidence.

EXPERIMENTAL OBSERVATION 5.4. All the examples listed in Conjectures 5.2 that are supposed to have real multiplication have the property that the endomorphism field is contained in the field of definition of the Picard group.

TABLE 2. Frequency of reduction to geometric Picard rank 22.

\begin{tabular}{lccccccc}
\hline \multirow{2}{*}{ Family } & \multicolumn{3}{c}{ \# relative frequency (in \%) of rank 22 per prime } \\
\cline { 2 - 4 } \cline { 7 - 8 } & \multicolumn{3}{c}{ Inert primes } & & \multicolumn{3}{c}{ Split primes } \\
\cline { 2 - 3 } \cline { 6 - 8 } & Min & Average & Max & & Min & Average & Max \\
\hline$V_{a}^{(2)}$ & 0.00 & 7.42 & 25.00 & & 0.00 & 6.14 & 25.00 \\
$V_{a}^{(5)}$ & 2.33 & 9.32 & 24.24 & & 0.00 & 5.84 & 16.00 \\
$V_{a, b}^{(2)}$ & 4.75 & 12.66 & 38.89 & & 1.50 & 7.39 & 22.73 \\
$V_{a, b}^{(3)}$ & 0.00 & 0.00 & 0.000 & & 2.33 & 7.40 & 20.59 \\
\hline
\end{tabular}




\section{Numerical evidence. Data for the real multiplication examples}

For each prime $p \in\{19, \ldots, 499\}$, we inspected all the possible specialisations of the families after reduction modulo $p$. Whenever this resulted in a non-singular surface, we computed the Weil polynomial and derived the geometric Picard rank. This confirmed properties 5.1(i) and (ii) in every case. In particular, only the Picard ranks 18 and 22 occur. Table 2 shows the statistics.

One might want to look more closely at the primes that result in reduction to geometric Picard rank 22 . In the case of the family $V_{a}^{(2)}, 77$ of 88 primes occur. The missing ones are $23,29,31,47,97,127,193,241,401,433$ and 449 . The reductions of these primes modulo 8 are $1^{6}, 5,7^{4}$. The only one inert in the endomorphism field $\mathbb{Q}(\sqrt{2})$ is 29 . For the family $V_{a, b}^{(2)}$, all 88 inspected primes occur, while for $V_{a}^{(5)} 87$ of the 88 primes occur and only 29 is missing. However, in the example $V_{a, b}^{(3)}$, only 44 of the 88 primes result in reductions to rank 22 and in fact these are precisely those splitting in the endomorphism field $\mathbb{Q}(\sqrt{3})$, a phenomenon for which we have no explanation.

Primes of reduction to rank 18. In the case of reduction to Picard rank 18, the transcendental factor split off the Weil polynomial is of degree 4. In our families, it was never a perfect power (cf. [42, Theorem 1.1]), but always turned out to be irreducible. It was either the norm of a quadratic polynomial $g \in E[t]$ over the endomorphism field or turned into a square under the operation of squaring all its roots. The first alternative occurred precisely at the primes split in $E$, while the second option came up at the inert primes.

\section{Numerical evidence. Data for the complex multiplication examples}

We report only on the numerical evidence for the three isolated examples. These are contained in the family $V_{\mathbf{a}, \mathbf{b}}^{(-1)}$. Thus, at least CM by $\mathbb{Q}(\sqrt{-1})$ is proven. As the surfaces have geometric Picard rank 16, the transcendental part of the cohomology is of relative dimension 1. Thus, one should expect phenomena that are very close to those known to occur for elliptic curves with complex multiplication.

To verify this, we run the point counting for all the primes below 1000. It turns out that all the reductions have geometric Picard rank either 16 or 22, where rank 16 appears exactly for the primes $1 \bmod 4$. A prime is ordinary if and only if it completely splits in the endomorphism field. Furthermore, the Frobenius eigenvalues on the transcendental part of $H_{\text {êt }}^{2}\left(V_{\overline{\mathbb{F}}_{p}}, \mathbb{Q}_{l}\right)$ are closely related to the endomorphism field $E$. More precisely, we have the following observations.

(a) In the case where $p$ completely splits in $E$, the eigenvalues of Frob are contained in $E$. They are of the form $\pm p(\bar{\pi} / \pi)$ for $(\pi)$ a prime above $p$. Note that the endomorphism fields have class number 1 , such that the ideals above $p$ are principal.

(b) In the case where $p$ splits into three primes, the characteristic polynomial of Frob on the transcendental lattice is $t^{6}-3 p^{2} t^{4}+3 p^{4} t^{2}-p^{6}=\left(t^{2}-p^{2}\right)^{3}$.

(c) In the case where $p$ splits into two primes, the characteristic polynomial of Frob on the transcendental lattice is of the form $t^{6}+a p^{2} t^{3}+p^{6}$, for $a \in \mathbb{Z}$ having the property that the discriminant of $t^{2}+a t+p^{2}$ is minus a square. Thus, the Weil polynomial of $X_{\mathbb{F}_{p^{3}}}$ is a cube of a quadratic polynomial having its roots in $\mathbb{Q}(i)$. These are of the form $p^{2} \varrho^{2}$, for $\varrho \in \mathbb{Q}(i)$ a prime above $p$. Let us note here once again that $\mathbb{Q}(i) \subset E$.

(d) In the case where $p$ is totally inert, the degree- 6 transcendental factor of the Weil polynomial is equal to $t^{6}-p^{6}$.

The complete computation took about 110 days of CPU time on one Intel Xeon E5-4650 processor running at $2.7 \mathrm{GHz}$.

Acknowledgements. We wish to thank David Harvey for valuable one-to-one talks, as well as the two anonymous referees for their suggestions on how to improve this note. 


\section{References}

1. T. G. Aввотt, K. S. Kedlaya and D. Roe, 'Bounding Picard numbers of surfaces using p-adic cohomology', Arithmetics, geometry, and coding theory (AGCT 2005), Séminaires et Congrès 21 (Société Mathématique de France, Paris, 2010) 125-159.

2. E. Artin, 'Quadratische Körper im Gebiete der höheren Kongruenzen II', Math. Z. 19 (1924) 207-246.

3. Z. I. Borevich and I. R. Shafarevich, Number theory (Academic Press, New York, 1966).

4. W. Bosma, J. Cannon and C. Playoust, 'The Magma algebra system I. The user language', J. Symbolic Comput. 24 (1997) 235-265.

5. F. Charles, 'On the Picard number of K3 surfaces over number fields', Algebra Number Theory 8 (2014) $1-17$.

6. T. Cormen, C. Leiserson and R. Rivest, Introduction to algorithms (MIT Press and McGraw-Hill, Cambridge, MA, and New York, 1990).

7. E. Costa, 'Effective computations of Hasse-Weil zeta functions', PhD Thesis, New York University, New York, 2015.

8. P. Deligne, 'La conjecture de Weil I', Publ. Math. Inst. Hautes Études Sci. 43 (1974) 273-307.

9. P. Deligne, (with J. F. de Boutot, A. Grothendieck, L. Illusie and J.-L. Verdier), Cohomologie Étale, Séminaire de Géométrie Algébrique du Bois Marie (SGA4 $\frac{1}{2}$ ), Lecture Notes in Mathematics 569 (Springer, Berlin, 1977).

10. P. Deligne, 'La conjecture de Weil II', Publ. Math. Inst. Hautes Études Sci. 52 (1980) 137-252.

11. P. Deligne and N. Katz, 'Groupes de monodromie en géométrie algébrique', Séminaire de Géométrie Algébrique du Bois Marie 1967-1969 (SGA 7), Lecture Notes in Mathematics 288, 340 (Springer, Berlin, 1973).

12. T. Diagana, Non-Archimedean linear operators and applications (Nova Science Publishers, New York, 2007).

13. B. M. Dwork, 'On the congruence properties of the zeta function of algebraic varieties', J. reine angew. Math. 203 (1960) 130-142.

14. B. M. Dwork, 'On the rationality of the zeta function of an algebraic variety', Amer. J. Math. 82 (1960) 631-648.

15. N. Elkies and A. Kumar, 'K3 surfaces and equations for Hilbert modular surfaces', Algebra Number Theory 8 (2014) 2297-2411.

16. A.-S. Elsenhans and J. JAhnel, 'K3 surfaces of Picard rank one and degree two', Algorithmic number theory (ANTS 8), Lecture Notes in Computer Science 5011 (Springer, Berlin, 2008) 212-225.

17. A.-S. Elsenhans and J. JAhnel, 'On Weil polynomials of K3 surfaces', Algorithmic number theory (ANTS 9), Lecture Notes in Computer Science 6197 (Springer, Berlin, 2010) 126-141.

18. A.-S. Elsenhans and J. Jahnel, 'The Picard group of a K3 surface and its reduction modulo p', Algebra Number Theory 5 (2011) 1027-1040.

19. A.-S. Elsenhans and J. Jahnel, 'Kummer surfaces and the computation of the Picard group', LMS J. Comput. Math. 15 (2012) 84-100.

20. A.-S. Elsenhans and J. JAhnel, 'Examples of K3 surfaces with real multiplication, in: Proceedings of ANTS 11', LMS J. Comput. Math. 17 (2014) 14-35.

21. A.-S. Elsenhans and J. Jahnel, 'On the characteristic polynomial of the Frobenius on étale cohomology', Duke Math. J. 164 (2015) 2161-2184.

22. O. Forster, Algorithmische Zahlentheorie (Vieweg, Braunschweig, 1996).

23. B. van GeEmen, 'Real multiplication on K3 surfaces and Kuga-Satake varieties', Michigan Math. J. 56 (2008) 375-399.

24. A. Grothendieck, (with I. De Bucur, C. Houzel, L. Illusie and J.-P. Serre), Cohomologie l-adique et fonctions L, Séminaire de Géométrie Algébrique du Bois Marie 1965-1966 (SGA 5), Lecture Notes in Mathematics 589 (Springer, Berlin, 1977).

25. R. Hartshorne, Algebraic geometry, Graduate Texts in Mathematics 52 (Springer, New York, 1977).

26. D. Harvey, 'Computing zeta functions of arithmetic schemes', Proc. Lond. Math. Soc. (3) 111 (2015) 1379-1401.

27. H. HASSE and E. WITT, 'Zyklische unverzweigte Erweiterungskörper vom Primzahlgrade $p$ über einem algebraischen Funktionenkörper der Charakteristik p', Monatsh. Math. Phys. 43 (1936) 477-492.

28. K. Kedlaya, 'Counting points on hyperelliptic curves using Monsky-Washnitzer cohomology', J. Ramanujan Math. Soc. 16 (2001) 323-338.

29. A. G. B. LAuder, 'A recursive method for computing zeta functions of varieties', LMS J. Comput. Math. 9 (2006) 222-269.

30. A. G. B. LAUder and D. WAN, 'Counting points on varieties over finite fields of small characteristic', Algorithmic number theory: lattices, number fields, curves and cryptography, MSRI Publications 44 (Cambridge University Press, Cambridge, 2008) 579-612.

31. R. van LUIJK, 'Rational points on K3 surfaces', PhD Thesis, University of California, Berkeley, 2005. 
32. R. VAN LUIJK, 'K3 surfaces with Picard number one and infinitely many rational points', Algebra Number Theory 1 (2007) 1-15.

33. L. Miller, 'The Hasse-Witt-matrix of special projective varieties', Pacific J. Math. 43 (1972) $443-455$.

34. S. PANCRATZ and J. Tuitman, 'Improvements to the deformation method for counting points on smooth projective hypersurfaces', Found. Comput. Math. 15 (2015) 1413-1464.

35. T. SAтон, 'The canonical lift of an ordinary elliptic curve over a finite field and its point counting', J. Ramanujan Math. Soc. 15 (2000) 247-270.

36. R. Schoof, 'Counting points on elliptic curves over finite fields in: Les Dix-huitièmes Journées Arithmétiques (Bordeaux 1993)', J. Théor. Nombres Bordeaux 7 (1995) 219-254.

37. S. G. TAnkeEv, 'Surfaces of K3 type over number fields and the Mumford-Tate conjecture', Izv. Akad. Nauk SSSR Ser. Mat. 54 (1990) 846-861 (Russian).

38. S. G. TANKeEv, 'Surfaces of K3 type over number fields and the Mumford-Tate conjecture II', Izv. Ross. Akad. Nauk Ser. Mat. 59 (1995) 179-206 (Russian).

39. D. WAN, 'Computing zeta functions over finite fields', Finite fields: theory, applications, and algorithms (Waterloo 1997), Contemporary Mathematics 225 (American Mathematical Society, Providence, RI, 1999) 131-141.

40. D. WAN, 'Algorithmic theory of zeta functions over finite fields', Algorithmic number theory: lattices, number fields, curves and cryptography, MSRI Publications 44 (Cambridge University Press, Cambridge, 2008) 551-578.

41. YU. G. Zarhin, 'Hodge groups of K3 surfaces', J. reine angew. Math. 341 (1983) 193-220.

42. Yu. G. Zarhin, 'Transcendental cycles on ordinary K3 surfaces over finite fields', Duke Math. J. 72 (1993) 65-83.

\section{Andreas-Stephan Elsenhans \\ Mathematisches Institut \\ Universität Paderborn \\ Warburger Straße 100 \\ D-33098 Paderborn \\ Germany}

elsenhan@math.uni-paderborn.de

\author{
Jörg Jahnel \\ Department Mathematik \\ Universität Siegen \\ Walter-Flex-Straße 3 \\ D-57068 Siegen \\ Germany
}

jahnel@mathematik.uni-siegen.de 